\title{
Accuracy of Clinical Coding for Procedures Performed in The ENT Ward Treatment Room
}

Katharine Hamlett, Rachel Edmiston, John de Carpentier Royal Preston Hospital, ENT Department

\section{Background}

Within ENT departments, many patients are often seen on the ward as ward attenders in 'treatment rooms'

A number of basic procedures can be carried out in these treatment rooms' e.g. FNE, nasal cautery, aural microsuction etc.

The Capita coding document in 2014 identified very poor levels of clinical coding within the $\mathrm{NHS}^{1}$

We identified that for all patients seen in our ward treatment room, only the 'ward attender' tariff (£68.41) was being paid to the ENT department - due to lack of awareness surround clinical coding

\section{A huge potential loss of income was highlighted}

\section{Objectives}

To improve the ENT junior doctors' engagement with clinical coding

To ensure that departmental funding reflects the procedural workload undertaken within the ENT treatment room

To improve treatment room documentation

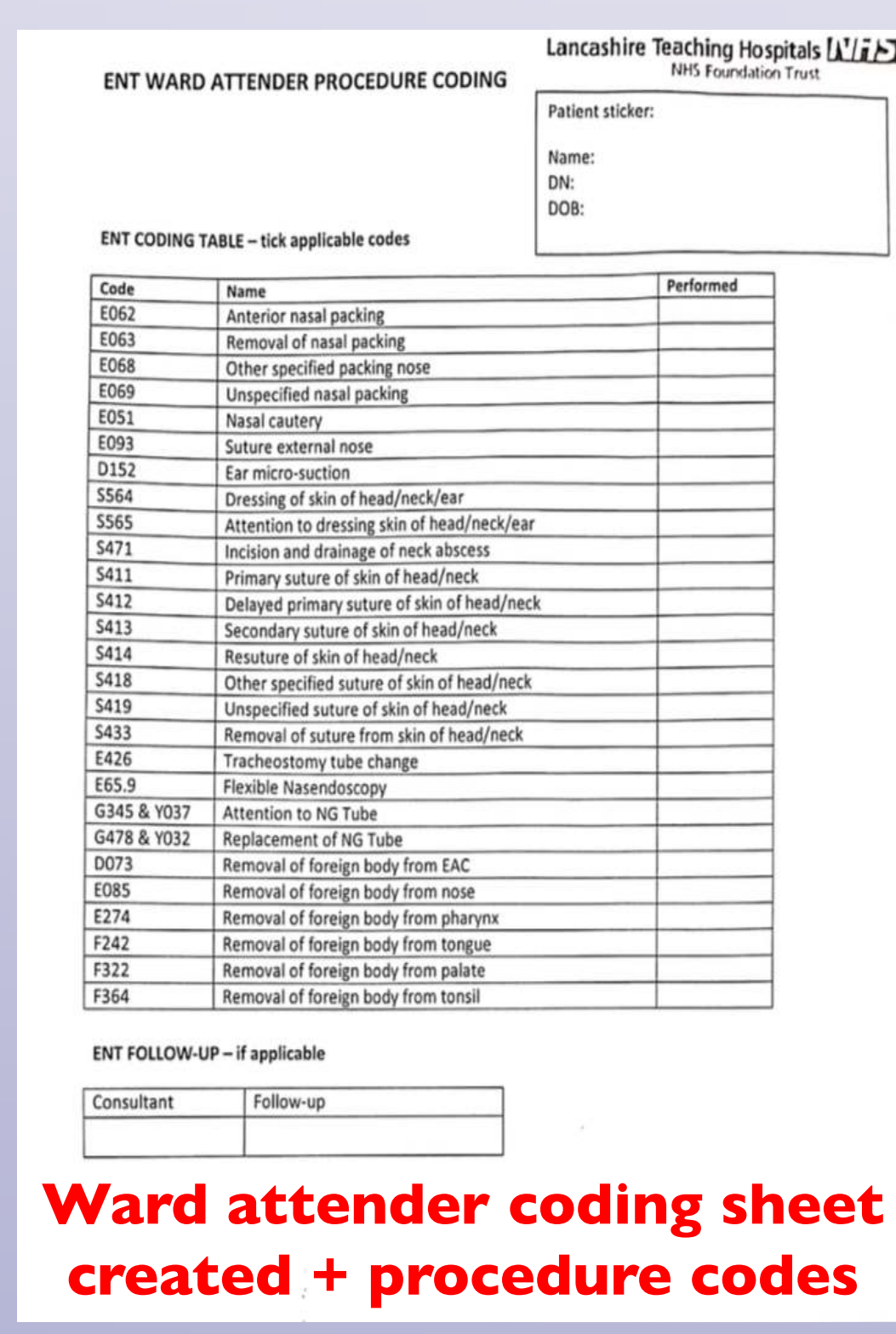

Methods

1) First audit cycle:

- 1 week data collection - all patients seen in treatment room

- All procedures recorded

- Procedure tariffs obtained - income loss calculated

- Presented at local audit meeting to raise coding awareness

2) Second audit cycle:

- Development of integrated coding/clerking proforma

- Introduction to team \& ward clerks

- 1 week data collection - all patients seen in treatment room

- All procedures recorded

- Procedure tariffs obtained - income loss calculated

- Figures presented to ENT business managers

\section{Results}

First audit: $£ 1716.71$ loss of income in 1 week (15 patients seen)

\section{Second audit: $£ 2532.60$ gain of income in 1} week (20 patients seen)

\section{1st audit cycle breakdown: (15 patients seen, 17 procedures) Ward Attender Tariff $=£ 68.41 \times 15=£ 1026.15$ \\ $3 \times$ Flexible nasal endoscopies $=£ 103.54 \quad \times 3=£ 310.62$ \\ $9 \times$ Micro-suction $=£ 88.01 \quad \times 9=£ 792.09$ \\ $1 \times$ Pope wick insertion $=£ 124.25 \times 1=£ 124.25$ \\ $1 \times$ Pope wick removal $=£ 117 \quad \times 1=£ 117$ \\ $3 \times 1 \& D$ head $\&$ neck abscess $=£ 124.25 \quad x 3=£ 372.75$ \\ Total money lost $=£ 1716.71$ \\ Estimate per month $=£ 6866.84$ \\ Estimate per year $=£ 82,402.08$ !! - potential revenue loss!}

2nd audit cycle breakdown: (20 patients seen, 24 procedures)

Ward Attender Tariff $=£ 68.41 \times 20=£ 1368.20$

$7 \times$ Flexible nasal endoscopies $=£ 103.54 \quad \times 7=£ 724.78$

$7 \times$ Micro-suction $=£ 88.01 \quad \times 7=£ 616.07$

$1 \times$ Dressing to head/neck $=£ 124.25 \times 1=£ 124.25$

$3 \times$ Attention to dressings $=£ 117 \times 3=£ 351.00$

$2 \times$ I\&D head and neck abscess $=£ 124.25 \quad \times 2=£ 248.50$

$3 \times$ Suturing of head / neck $=£ 117.00 \times 3=£ 351.00$

$1 \times$ Removal of sutures $=£ 117.00 \quad \times 1=£ 117.00$

Total money gained by accurate coding $=£ 2532.60 ! !$

Estimate per year gained $=£ 131,695.20$ !

\section{$100 \%$ SHO engagement with using clerking \& coding proformas}

\section{Conclusions}

This easily replicable closed-loop audit was able to hugely improve the yearly income for our ENT department to ensure that the funding reflects the departmental workload

Clinical coding should be recognised for its importance in ensuring that departmental performance reflects the budget it receives.

Additional funding can help to improve patient care 